\title{
A Novel Transient Simulation for 3-D Multilevel Interconnections on Complex Topography
}

\author{
Hsin-Ming Hou, Student Member, IEEE, Chin-Shown Sheen, Student Member, IEEE, and Ching-Yuan Wu, Member, IEEE
}

\begin{abstract}
An efficient method is presented to model the transient characteristics of distributed resistor-capacitor of ULSI multilevel interconnections on complex topography, in which the reformulation of the boundary-element method (BEM) and the Padè-via-Lanczos (PVL) algorithm associated with multilayer Green's function can avoid the redundant works on both volume mesh and transient analysis associated with the finite-difference method. An adaptive multilayer Green's function is adopted to investigate several cases that have revealed interesting physical mechanisms in charge transfer between conductors on multilayer topography. To improve the timing analysis efficiency of the finite-difference method, the dominant poles are obtained by introducing the PVL algorithm for model-order reduction. Hence, it is easy to calculate the transient characteristics of both parallel conductors and complicated configurations such as crossing lines, corners, contacts, multilayers, and their combinations.
\end{abstract}

Index Terms - Boundary-element method, Green's function, multilevel interconnection, Padè-via-Lanczos algorithm, ULSI.

\section{INTRODUCTION}

$\mathbf{T}$ HE IC interconnection delay modeled with high accuracy in ULSI circuits are becoming increasingly important and necessary. The geometry of the interconnection is an important factor in the transmission behavior and layoutto-circuit extractors to obtain accuracy and efficiency. In the past, the interconnections are subdivided into small elements and each element is replaced by a lumped RC-section. However, the number of elements must be sufficiently large to guarantee that the distributed properties of the interconnection are accurately reflected by the resulting network. It was suggested that simulations with sufficient accuracy based on asymptotic waveform evaluation (AWE) [1] could be performed by computing the transient characteristics of the electric fields both inside and outside the interconnects by the finite-difference discretization of Laplace equation [2]. However, this method is time-consuming and the illconditioned problem of matching the moments for the AWE method will explicitly happen. Recently, the boundary-element approach (BEM) based on Green's theorem, which has been widely applied to important issues of interconnections but can avoid the volume mesh associated with the finite-difference methods, has been investigated by many research groups [3], [4]. To deal with these problems, an efficient and accurate

Manuscript received April 23, 1998. The review of this paper was arranged by Editor M. Fukuma. This work was supported by the National Science Council, Taiwan, R.O.C., under Contract NSC88-2215-E009-039.

The authors are with the Advanced Semiconductor Device Research Laboratory and the Institute of Electronics, National Chiao-Tung University, Hsinchu 300, Taiwan, R.O.C.

Publisher Item Identifier S 0018-9383(99)02385-0. modeling of transient characteristics of different dielectric layers for such a densely packed multiconductor system has been developed.

Our work uses an adaptive multilayer Green's function for BEM to examine the voltage and current responses of multilevel conductor system. After the Laplace transformation of the large linear equations, all the poles and residues can be obtained by diagonalization of matrix. To improve timing analysis efficiency, the Padè-via-Lanczos (PVL) algorithm [5] is introduced to generate arbitrary numbers of poles and residues with little numerical degradation. Besides, this method can avoid the ill-conditioned problem of matching the moments induced explicitly by the AWE method. At the same time, we find that the transient characteristics of the interconnection strongly depend on its capacitance obtained by [3] for steady state. Major improvements are the reformulation of the BEM and PVL algorithm associated with multilayer Green's function to model the transient characteristics, which are proven to be applicable to even more complex configuration.

\section{MODEL DEVELOPMENT}

\section{A. Green's Formulation}

It is adequate to assume that the relaxation time of the volume charge is short enough to be negligible so that we only consider the transient response of surface charge. The BEM for calculating multiconductor transient characteristics is based on a Green's function approach to the electrostatic problem. To consider a geometry with a number of conductors embedded in a perfectly stratified dielectric medium shown in Fig. 1, the multilayer Green's function $G\left(x, x_{s}\right)$ is used [3], where $x$ is a view point and $x_{s}$ is a source point. For the wavelength of electromagnetic wave longer than the length of conductor, the magnetic fields can be negligible. From the Laplace equation $\nabla^{2} V \equiv 0$ and its Green's function defined as $\nabla^{2} G \equiv-\delta / \varepsilon\left(x_{s}\right)$, one can obtain the time-dependent boundary-integral equation by the conservation of charges [4]

$$
\begin{aligned}
\tau(x) \frac{\partial V(x)}{\partial t}= & c(x) V(x)+\int_{S} V\left(x_{s}\right) \varepsilon\left(x_{s}\right) \nabla_{x_{s}} G\left(x, x_{s}\right) \cdot d \vec{S} \\
& +\int_{S_{c}} G\left(x, x_{c}\right) \tau\left(x_{c}\right) J_{\text {external }}\left(x_{c}\right) d S_{c}
\end{aligned}
$$

where $\tau=\varepsilon / \sigma$ is the dielectric relaxation time and $S_{c}$ denotes contact region.

After discretization of (1), the surface of conductors is divided into $N$ elements and the meshing number of contact 


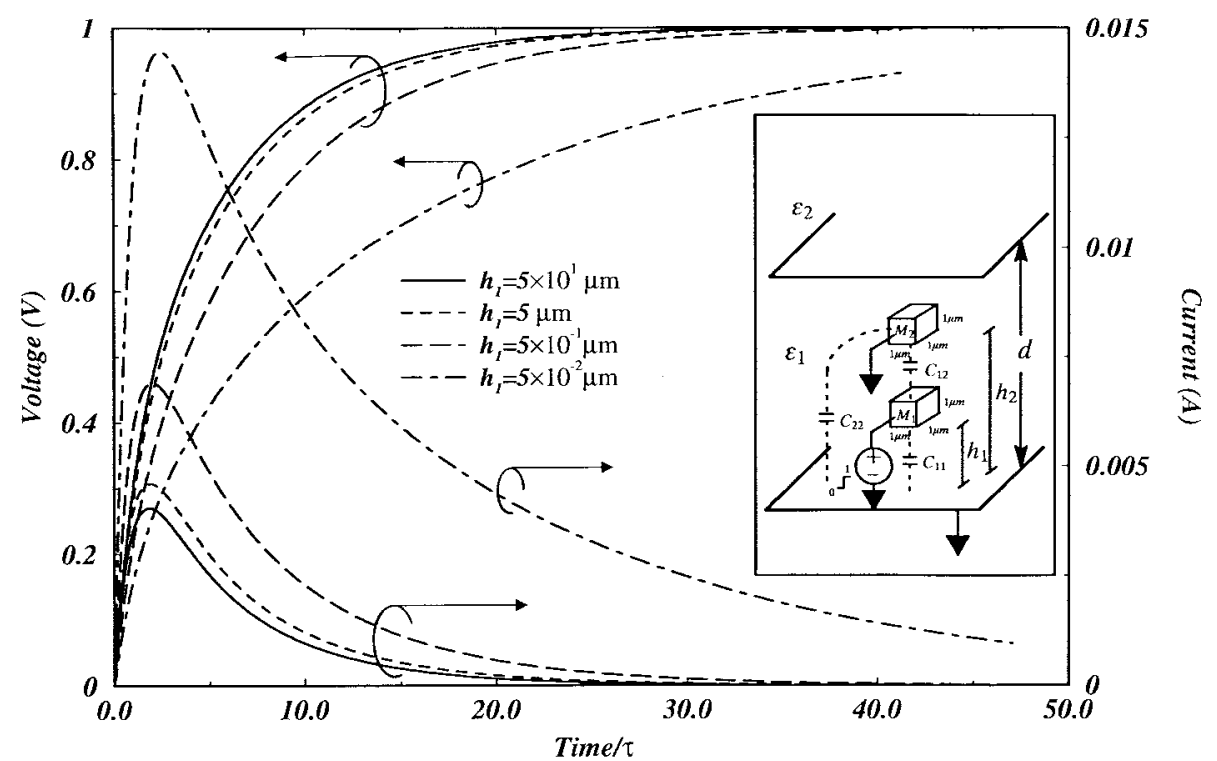

Fig. 1. The inset figure shows the configuration of multilevel interconnections ( $\rho_{\text {polysilicon }}=0.01 \Omega-\mathrm{cm}$ ) in inhomogeneous medium over an infinite ground plane and its equivalent circuit. And the transient characteristics for the end of the conductor $M_{1}$ versus different vertical positions of the conductor $M_{1}$ in homogeneous medium over the ground plane $\left(\varepsilon_{1}=\varepsilon_{2}=3.9 \varepsilon_{0}, h_{2}=2.5 \mu \mathrm{m}\right.$, and the total number of nodes $\left.=216\right)$.

part of conductor surface is $M$. The Galerkin method applied to the discretized boundary integral equation. Equation (1) can be rewritten as

$$
\begin{aligned}
& \tau\left(x_{i}\right) \frac{\partial V\left(x_{i}\right)}{\partial t} \\
& \quad=\frac{1}{2} V\left(x_{i}\right)+\sum_{j=1}^{N} \int V\left(x_{j}\right) G_{\mathrm{ij}}^{(n)}+\sum_{j=1}^{M} \rho_{j}\left(x_{j}\right) G_{\mathrm{ij}}
\end{aligned}
$$

where $\rho\left(x_{c}\right)=\tau\left(x_{c}\right) J\left(x_{c}\right)$, the matrix elements $G_{\mathrm{ij}}=\int_{S_{j}}$ $G\left(x_{i}, x_{j}\right) f_{j}\left(x_{j}\right) d S_{j}, G_{\mathrm{ij}}^{(n)}=\int_{S_{j}} \varepsilon\left(x_{j}\right) \nabla_{x_{j}} G\left(x_{i}, x_{j}\right) f_{j}\left(x_{j}\right) \cdot d \overrightarrow{S_{j}}$ and $f_{j}\left(x_{j}\right)$ is the constant-element shape function assigned to the boundary element $S_{j}$ such that $\int_{S_{j}} f_{j}\left(x_{j}\right) d S_{j}=1$.

To obtain a concise form in matrix notation for the discretized boundary integral formulation, the above equation can be reformulated as

$$
\tau\left[\frac{\partial V}{\partial t}\right]=\left[H_{\mathrm{ij}}\right][V]+\left[G_{\mathrm{ij}}\right][\rho]
$$

where $\left[H_{\mathrm{ij} j}\right]=\frac{1}{2}[I]+\left[G_{\mathrm{ij}}^{(n)}\right]$. Explicitly, $[V]=\left[V_{1}, V_{2}, \ldots, V_{N}\right]^{T}$ and $[\rho]=\left[\rho_{1}, \rho_{2}, \cdots, \rho_{M}\right]^{T}$ are the potentials and charges of element, respectively; $[I]$ is the identity matrix. After the Laplace transformation of the above equation, one can obtain the following expression:

$$
\tau s\left[\begin{array}{l}
V_{c} \\
V_{f}
\end{array}\right]=\left[\begin{array}{ll}
H_{\mathrm{cc}} & H_{\mathrm{cf}} \\
H_{\mathrm{fc}} & H_{\mathrm{ff}}
\end{array}\right]\left[\begin{array}{c}
V_{c} \\
V_{f}
\end{array}\right]+\left[\begin{array}{c}
G_{\mathrm{cc}} \\
G_{\mathrm{fc}}
\end{array}\right]\left[\rho_{c}\right]
$$

where the index " $c$ " denotes contact region and " $f$ " denotes floating region. Define the Laplace transformation of the input excitation vector $\left[V_{c}\right]$ as $[b] u(s)$, we can obtain the contact charges of conductors as

$$
\begin{aligned}
{\left[\rho_{c}\right]=} & \tau s\left[G_{\mathrm{Cc}}\right]^{-1}[b] u(s)-\left[G_{\mathrm{Cc}}\right]^{-1}\left[H_{\mathrm{cc}}\right][b] u(s) \\
& -\left[G_{\mathrm{Cc}}\right]^{-1}\left[H_{\mathrm{cf}}\right]\left[V_{f}\right]
\end{aligned}
$$

and the potential of floating region on conductor as

$$
\left[V_{f}\right]=\frac{\left[b_{2}\right] u(s)}{\tau}+(\tau s[I]-[A])^{-1}[v] u(s)
$$

where $[A]=\left[H_{\mathrm{ff}}\right]-\left[B_{\mathrm{fc}}\right]\left[H_{\mathrm{cf}}\right],\left[b_{1}\right]=\left(\left[H_{\mathrm{fc}}\right]-\left[B_{\mathrm{fc}}\right]\left[H_{\mathrm{cc}}\right]\right)[b]$, $\left[b_{2}\right]=\tau\left[B_{\mathrm{fc}}\right][b],\left[B_{\mathrm{fc}}\right]=\left[G_{\mathrm{fc}}\right]\left[G_{\mathrm{cc}}\right]^{-1}$, and $[v]=\left[b_{1}\right]+$ $[A]\left[B_{\mathrm{fc}}\right][b]$. To obtain transient behavior, the transfer function for voltage is defined as

$$
H(s)=\frac{[l]^{T}\left[V_{f}(s)\right]}{u(s)}
$$

where $[l]^{T} \in R^{N-M}$ is the vector which picks out the voltages from a specific observation point. By combining the above two equations, we immediately obtain

$$
H(s)=k+[l]^{T}(\tau s[I]-[A])^{-1}[v]
$$

where $k=[l]^{T}\left[b_{2}\right] / \tau$. Besides the transfer function for current can be defined as

$$
H(s)=\frac{[L]^{T}\left[J_{\text {external }}\left(x_{c}, s\right)\right]}{u(s)}
$$

where $[L]^{T} \in R^{M}$ is the vector which picks out the currents from a specific observation point. By combining (5) and the above equation, we also get

$$
H(s)=k_{0}+s k_{1}-\frac{1}{\tau}[L]^{T}\left[G_{\mathrm{cc}}\right]^{-1}\left[H_{\mathrm{cf}}\right](\tau s[I]-[A])^{-1}[v]
$$

where $k_{0}=-(1 / \tau)[L]^{T}\left[G_{\mathrm{CC}}\right]^{-1}\left[H_{\mathrm{cc}}\right][b]-\left(1 / \tau^{2}\right)[L]^{T}\left[G_{\mathrm{Cc}}\right]^{-1}$ $\left[H_{\mathrm{cf}}\right]\left[b_{2}\right]$ and $k_{1}=[L]^{T}\left[G_{\mathrm{cc}}\right]^{-1}[b]$.

\section{B. Padè-via-Lanczos Algorithm}

Using the Padè-via-Lanczos (PVL) algorithm [5], the reduced-order transfer function for (8) can then be constructed as

$$
H_{q}(s)=k+[l]^{T}[v]\left[e_{1}\right]^{T}\left(\tau s[I]-\left[T_{q}(s)\right]\right)^{-1}\left[e_{1}\right]
$$


which is just the $q$ th Padè approximation of $H$. Whereas $\left[e_{1}\right]=\left[\begin{array}{lllll}1 & 0 & 0 & \cdots & 0\end{array}\right]^{T} \in R^{q},\left[T_{q}(s)\right]$ is the tridiagonal matrix and $q \times q$ upper Hessenberg matrix. The transfer function of the reduced $q$ th-order system can be obtained directly by using the eigen-decomposition $\left[S_{q}\right]\left[\operatorname{diag}\left(\lambda_{1}, \lambda_{2}, \cdots, \lambda_{q}\right)\right]\left[S_{q}\right]^{-1}$. After manipulation, we can obtain

$$
H_{q}(s)=k+\sum_{j=1}^{q} \frac{R_{j}}{s-P_{j}}
$$

where $[\mu]^{T}=\left[e_{1}\right]^{T}\left[S_{q}\right]$ and $[\nu]=\left[S_{q}\right]^{-1}\left[e_{1}\right]$. The $j$ th pole and the $j$ th residue of $H_{q}(s)$ are $P_{j}=\lambda_{j} / \tau$ and $R_{j}=[l]^{T}[v] \mu_{j} \nu_{j} / \tau$, respectively. Similarly, the reduced-order transfer function for (10) can be also derived by the PVL algorithm.

\section{Multilayer Green's Function}

The derivations given in the above sections can be used when there is the multilayer region. Generally, the problem of an appropriate three-dimensional (3-D) Green's function in multilayer regions can be obtained by two different methods: one is the method of images [8]; the other is the Fourier integral techniques [3], [7] to obtain the Green's function. Comparatively, the latter method is less cumbersome and more physical.

We consider that the interconnections are placed in a stratified medium over an electric ground plane. This assumption is valid when the substrate is heavily doped and the IC is reasonably planar. So, the $\mathrm{Si}$-substrate is the infinite ground plane and the $\mathrm{SiO}_{2}$ layer is used as protective material. We use the Fourier integral technique to obtain the Green's function for $\mathrm{Si}-\mathrm{SiO}_{2}$ system shown in Fig. 1. When both the field point and the source point are in the same protective coating such as oxide, the Green's function for stratified medium can be written as

$$
\begin{aligned}
& G(p, q) \\
& =\frac{1}{4 \pi \varepsilon_{1}}\left\{\frac{1}{\sqrt{\left(x-x^{\prime}\right)^{2}+\left(y-y^{\prime}\right)^{2}+\left(z-z^{\prime}\right)^{2}}}\right. \\
& -\frac{1}{\sqrt{\left(x-x^{\prime}\right)^{2}+\left(y-y^{\prime}\right)^{2}+\left(z+z^{\prime}\right)^{2}}} \\
& +\sum_{n=0}^{\infty}(-1)^{n}\left(\frac{\varepsilon_{1}-\varepsilon_{2}}{\varepsilon_{1}+\varepsilon_{2}}\right)^{(n+1)} \\
& \cdot\left[\frac{1}{\sqrt{\left(x-x^{\prime}\right)^{2}+\left(y-y^{\prime}\right)^{2}+\left[2(n+1) d-\left(z+z^{\prime}\right)\right]^{2}}}\right. \\
& -\frac{1}{\sqrt{\left(x-x^{\prime}\right)^{2}+\left(y-y^{\prime}\right)^{2}+\left[2(n+1) d+\left(z-z^{\prime}\right)\right]^{2}}} \\
& +\frac{1}{\sqrt{\left(x-x^{\prime}\right)^{2}+\left(y-y^{\prime}\right)^{2}+\left[2(n+1) d+\left(z+z^{\prime}\right)\right]^{2}}} \\
& \left.\left.-\frac{1}{\sqrt{\left(x-x^{\prime}\right)^{2}+\left(y-y^{\prime}\right)^{2}+\left[2(n+1) d-\left(z-z^{\prime}\right)\right]^{2}}}\right]\right\}
\end{aligned}
$$

where $\varepsilon_{1}$ denotes the dielectric constant of $\mathrm{SiO}_{2} ; \varepsilon_{2}$ denotes the permittivity of vacuum or $\mathrm{Si}_{3} \mathrm{~N}_{4}$; and $d$ is the thickness of $\mathrm{SiO}_{2}$. The source point $q\left(x^{\prime}, y^{\prime}, z^{\prime}\right)$ and the field point $p(x, y, z)$ are space variables, which are described in the rectangular coordinate shown in Fig. 1. In (13), the physical meaning of potential can be divided into three terms: the first term is due to the contribution from the uniform dielectric $\varepsilon_{1}$; the second term can be considered as the contribution from the infinite ground plane; while the third term represents the contribution from different dielectrics.

\section{ELEMENT INTEGRATIONS}

The constant flat boundary element system is used here. The boundary $\Gamma$ is approximated by a polyhedron of $N$ flat sides, i.e., $\Gamma=\Gamma_{1}+\Gamma_{2}+\cdots+\Gamma_{N}$. Here we choose the triangular element, so $N=3$. The integral calculations of matrix elements $G_{\mathrm{ij}}$ and $G_{\mathrm{ij}}^{(n)}$ in (2) are the most critical part of a simulator, since it determines the computing time and the accuracy. For the constant boundary element, the integral terms of matrix elements, $G_{\mathrm{ij}}$ and $G_{\mathrm{ij}}^{(n)}$, are in analytical forms, which can be expressed as [6]

$$
\begin{aligned}
G_{\mathrm{ij}}^{(n)}= & \sum_{\Gamma_{1}}^{\Gamma_{3}}\left[\tan ^{-1}(Z Y)+\operatorname{sign}(Z) \Delta \Theta\right] \\
G_{\mathrm{ij}}= & \frac{1}{2 \varepsilon\left(x_{j}\right) A_{j}} \sum_{\Gamma_{1}}^{\Gamma_{3}}\left[D \ln \left|\frac{r_{1}+r_{2}+L}{r_{1}+r_{2}-L}\right|\right. \\
& \left.-|Z| \tan ^{-1}(|Z| Y)+|Z| \Delta \Theta\right]
\end{aligned}
$$

where $Y=D\left(r_{1} l_{2}-r_{2} l_{1}\right) / D_{1}^{2} r_{1} r_{2}+z^{2} l_{1} l_{2}$ and

$$
\Delta \Theta= \begin{cases}(a) & \text { corner angle } \\ (b) & 2 \pi \\ (c) & 0\end{cases}
$$

in which the condition (a) in (16) is the projection point on vertex; the condition (b) is the projection point on boundary or inside triangular element; and the condition (c) is else. It's important to note that the $\Delta \Theta$ in the analytical integral solution is an effective value and is not an actual geometrical angle. The parameters of $Z, D, r_{1}, r_{2}, l_{1}, l_{2}$, and $L$ are shown in Fig. 2; the parameter of $A_{j}$ is the area of the $j$ th triangular element; the term $\tan ^{-1}(y / x)$ in (14) is defined to take a value in $(-\pi, \pi)$ as the counterclockwise angle from $x$-axis in $x y$-plane. By using multilayer Green's functions, each matrix element is consisted of infinite series of surface integral. For the constant boundary element, the integral terms of matrix elements are in analytical forms.

According to the layout data, an automatic mesh generation takes place, in which node numbers and their coordinates are linked to the pre-processor of boundary element tools. Hence, it has been pointed out [3] that the calculated capacitance values are sensitive with respect to selection of nodes. To examine the accuracy and the efficiency of the sinusoidal weighting method, we employ the following scheme within each other of vertex points, $\vec{r}_{i, v e r t e x 1}$ and $\vec{r}_{i, v e r t e x 2}$, in which one takes

$$
\begin{aligned}
& \vec{r}_{i, k}=\left(\frac{\vec{r}_{i, \text { vertex } 2}+\vec{r}_{i, \text { vertex } 1}}{2}\right)-\left(\frac{\vec{r}_{i, \text { vertex } 2}-\vec{r}_{i, \text { vertex } 1}}{2}\right) \\
& \times\left(\cos \frac{(k-1) \pi}{K}\right)^{n}
\end{aligned}
$$




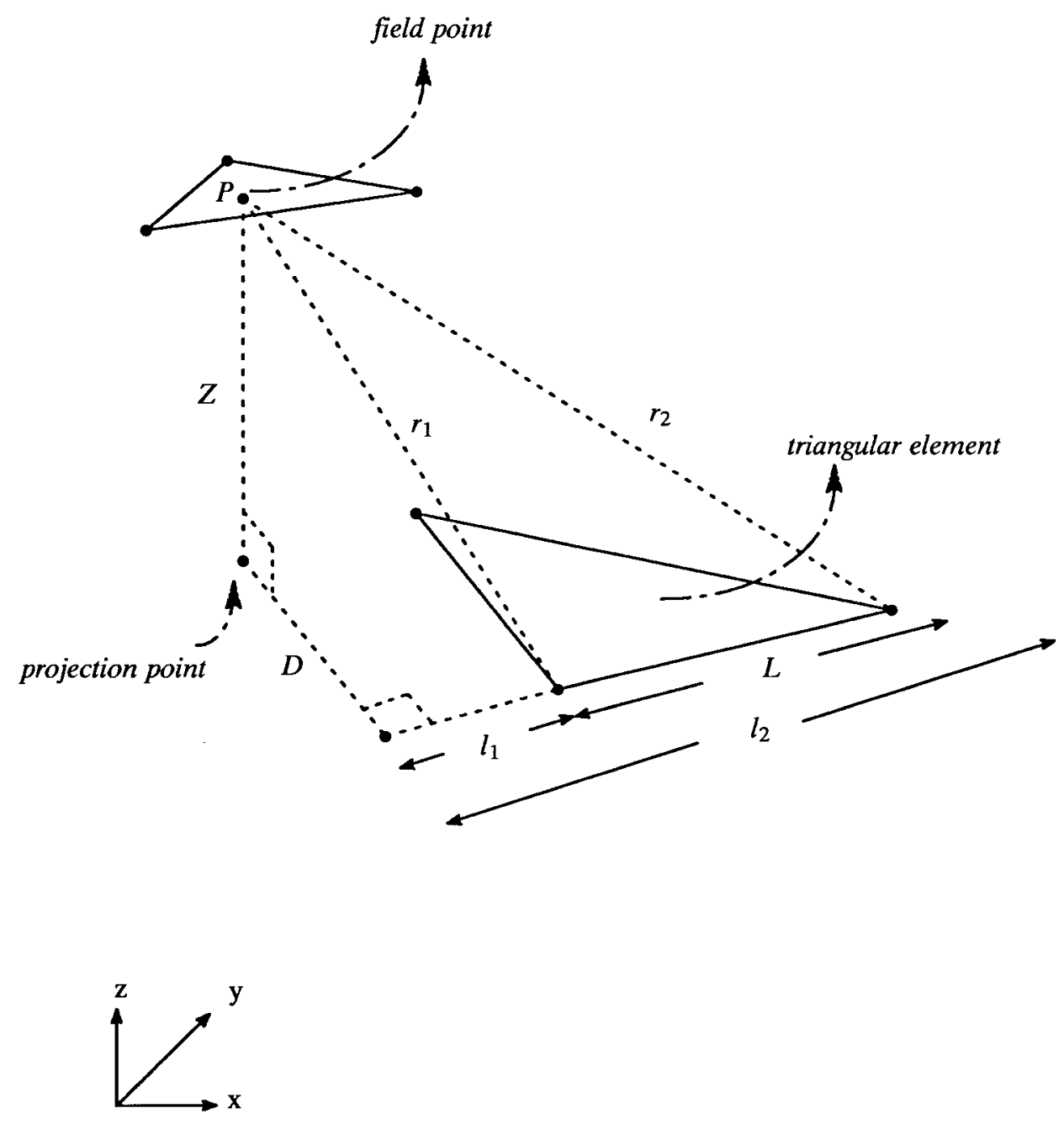

Fig. 2. Configuration of the field point and the triangular element based on three source points.

where $k=1,2, \cdots, K ; K$ is the total number of nodes between vertices and is a function of the three independent coordinate variables in 3-D space; $\vec{r}_{i, k}$ is the position for the $k$ th node of the $i$ th conductor; and $n$ is the weighting factor [3]. In general, as $n$ is smaller than one, the selective nodes are closer to the vertex.

\section{RESULTS AND COMPARISONS}

The most obvious application of our proposed method is to determine how much changes in voltages on a given conductor are capacitively transmitted to nearby conductor in inhomogeneous dielectric material oriented over an infinite ground plane. For the general case, we consider the scenario described in Fig. 1, in which a voltage step is applied to the near end of $M_{1}$ conductor. Actually, when the pumping charges are driven to one end of conductor, according to the image charges induced by the multiconductors on multilayer topography we adopted, the coupling capacitance of interconnections becomes complicated to analyze. The voltage responses at both the far ends of the parallel conductors are monitored, in which $M_{2}$ has its near end grounded. For this configuration, typically one end of every conductor is driven and the other end is connected to a high-impedance input, which can be considered as open.
For the convenience of monitoring, all the values in the tables but not all the figures listed are calculated at far end of $M_{1}$ conductor. The accuracy of the proposed method could be checked against data in Table I for two parallel conductors and homogeneous dielectric material oriented over an infinite ground plane shown in Fig. 1. One can see from Table I that the relative error of dominant pole will converge to less than about $6 \%$ for the number of nodes larger than 216 under equal-space meshing and the CPU time will be less than $156 \mathrm{~s}$. To investigate the effectiveness of the sinusoidal weighting scheme, which has been proven to be efficient [3], we calculate the dominant poles for Fig. 1 under different grid-partition conditions, as shown in Table II. The second and third rows are calculated under equal-space meshing as references for other sinusoidal weighting conditions. From Table II, it can be found that the relative error will grow as $n$ value is both larger and less than one. Obviously, the sinusoidal weighting method for the transient cases has no particular advantage as compared to the steady-state case [3], due to the complex charge pumping process. It should be noted that the computing time is made on a HP-735 workstation, which depends not only on the algorithm presented here but also on the method of grid partition. Furthermore, to exam 
TABLE I

The Accuracy and the CPU Time Versus the Total Mesh Number for THE INSET OF FIG. 1. $\left(\varepsilon_{1}=\varepsilon_{2}=3.9 \varepsilon_{0}, h_{1}=0.5 \mu \mathrm{m}\right.$, AND $\left.h_{2}=2.5 \mu \mathrm{m}\right)$

\begin{tabular}{|c|c|c|c|c|c|c|}
\hline $\begin{array}{l}\text { Number } \\
\text { of nodes }\end{array}$ & K & $\begin{array}{c}\text { Dominant } \\
\text { pole }(P) \times 10^{13}\end{array}$ & $\begin{array}{c}\text { Residue (R) } \\
\times 10^{13} \\
\end{array}$ & $\begin{array}{l}\text { P-Relative } \\
\text { error (\%) }\end{array}$ & $\begin{array}{l}R-\text { Relative } \\
\text { error (\%) }\end{array}$ & $\begin{array}{l}\text { CPU time } \\
\text { (in sec) }\end{array}$ \\
\hline 24 & $1,1,1$ & -5.146557 & 3.885458 & 42.22 & 45.06 & 1.1 \\
\hline 64 & $2,2,1$ & -4.303452 & 3.238126 & 18.92 & 20.90 & 9.0 \\
\hline 96 & $2,2,2$ & -4.139249 & 2.843728 & 14.38 & 6.171 & 22.0 \\
\hline 120 & $3,3,1$ & -4.107363 & 3.141295 & 13.50 & 17.28 & 40.0 \\
\hline 192 & $4,4,1$ & -4.029040 & 3.117062 & 11.34 & 16.38 & 125.4 \\
\hline 216 & $3,3,3$ & -3.838243 & 2.710518 & 6.064 & 1.197 & 155.2 \\
\hline 360 & $3,3,6$ & -3.785058 & 2.918285 & 4.594 & 8.954 & 536.1 \\
\hline 384 & $4,4,4$ & -3.698615 & 2.696808 & 2.205 & 0.6853 & 694.3 \\
\hline 600 & $5,5,5$ & -3.618806 & 2.678452 & 0.0 & 0.0 & 2414.8 \\
\hline
\end{tabular}

TABLE II

The Effectiveness of the Sinusoidal Weighting Scheme FOR THE INSET OF FIG. 1. $\left(\varepsilon_{1}=\varepsilon_{2}=3.9 \varepsilon_{0}, h_{1}=0.5 \mu \mathrm{m}\right.$, $h_{2}=2.5 \mu \mathrm{m}$, AND THE TOTAL NumBer OF NODES $=216$ )

\begin{tabular}{c|c|c|c|c|c|c|c}
\hline $\begin{array}{c}\text { Number } \\
\text { of nodes }\end{array}$ & $K$ & $n$ & $\begin{array}{c}\text { Dominant } \\
\text { pole }(P) \times 10^{13}\end{array}$ & $\begin{array}{c}\text { Residue }(R) \\
\times 10^{13}\end{array}$ & $\begin{array}{c}P \text {-Relative } \\
\text { error (\%) }\end{array}$ & $\begin{array}{c}\text { R-Relative } \\
\text { error (\%) }\end{array}$ & $\begin{array}{c}C P U \\
\text { iime (sec) }\end{array}$ \\
\hline \hline 600 & $5,5,5$ & & -3.618806 & 2.678452 & 0.0 & 0.0 & 2414.8 \\
\hline 216 & $3,3,3$ & -3.838243 & 2.710518 & 6.064 & 1.197 & 155.2 \\
\hline 216 & $3,3,3$ & 0.1 & -4.699800 & 5.148348 & 29.87 & 92.21 & 160.6 \\
\hline 216 & $3,3,3$ & 0.3 & -4.247978 & 3.820603 & 17.39 & 42.64 & 161.0 \\
\hline 216 & $3,3,3$ & 0.5 & -4.023868 & 3.295597 & 11.19 & 23.04 & 163.4 \\
\hline 216 & $3,3,3$ & 0.7 & -3.905898 & 3.026501 & 7.933 & 12.99 & 160.4 \\
\hline 216 & $3,3,3$ & 0.9 & -3.846942 & 2.875552 & 6.304 & 7.359 & 165.0 \\
\hline 216 & $3,3,3$ & 1 & -3.831677 & 2.826257 & 5.882 & 5.518 & 163.8 \\
\hline 216 & $3,3,3$ & 3 & -3.991188 & 2.756598 & 10.29 & 0.2918 & 151.5 \\
\hline 216 & $3,3,3$ & 5 & -4.099443 & 2.820993 & 13.28 & 5.322 & 149.9 \\
\hline 216 & $3,3,3$ & 7 & -4.129329 & 2.817577 & 14.11 & 5.194 & 139.8 \\
\hline 216 & $\mathbf{3 , 3 , 3}$ & 9 & -4.136917 & 2.843510 & 14.32 & 6.162 & 140.6 \\
\hline
\end{tabular}

the efficiency of our proposed model, the relative errors are demonstrated for different number of poles. From Table III, as the number of computed poles $q$ is small increased, one can see the relative error is greatly reduced in order of magnitude. At the same time, the $H_{q}$ approximation form is more and more approaching the exact frequency response $H$. It is worth noting that the relative error for $q=8$ is only about $0.008383 \%$ and the CPU time is greatly reduced to about half of the value in the last row with $q=180$.

To illustrate the flexibility of our proposed technique, we consider more general cases for different values of the ground plane position and the dielectric constant for the region 2 . To brief the article, the major physical meanings are extracted from the transient characteristics and listed in Table IV, which monitor the far ends of the $M_{1}$ and $M_{2}$ conductors, respectively. From rows 3-6 of Table IV, one can see that as the height $h_{1}$ increases exponentially from 0.05 to $50 \mu \mathrm{m}$, the current peak for $M_{1}$ decreases almost inverse proportion and
TABLE III

The EfFectiveness OF THE PVL Algorithm FOR THE INSET OF FIG. 1. $\left(\varepsilon_{1}=\varepsilon_{2}=3.9 \varepsilon_{0}, h_{1}=0.5 \mu \mathrm{m}\right.$, $h_{2}=2.5 \mu \mathrm{m}$, AND THE TOTAL NUMBer OF NODES $\left.=216\right)$

\begin{tabular}{c|c|c|c|c|c|c}
\hline $\begin{array}{c}\text { Number } \\
\text { of nodes }\end{array}$ & $q$ & $\begin{array}{c}\text { Dominant } \\
\text { pole }(P) \times 10^{33}\end{array}$ & $\begin{array}{c}\text { Residue }(R) \\
\times 10^{13}\end{array}$ & $\begin{array}{c}\text { P-Relative } \\
\text { error }(\%)\end{array}$ & $\begin{array}{c}R-\text { Relative } \\
\text { error }(\%)\end{array}$ & $\begin{array}{c}\text { CPU time } \\
(\text { in sec })\end{array}$ \\
\hline \hline 216 & 1 & -4.150683 & 3.294246 & 8.140 & 21.54 & 73.6 \\
\hline 216 & 3 & -3.978197 & 3.281191 & 3.646 & 21.05 & 72.3 \\
\hline 216 & 6 & -3.845714 & 2.775197 & 0.1946 & 2.386 & 72.5 \\
\hline 216 & 8 & -3.838563 & 2.713568 & 0.008337 & 0.1125 & 72.7 \\
\hline 216 & 180 & -3.838243 & 2.710518 & 0.0 & 0.0 & 155.2 \\
\hline
\end{tabular}

TABLE IV

Comparisons of Transient Characteristics for DifFerent Vertical Positions of the Conductor $M_{1}$ IN Homogeneous Medium Over the Ground Plane and Different Permitivities of Inhomogeneous Dielectric Material Oriented Over an Infinite Ground Plane (The Total Number of Nodes $=216)$ Shown IN the InSET OF Fig. 1

\begin{tabular}{|c|c|c|c|c|c|}
\hline & & & & & $M_{2}$ \\
\hline & & & $\begin{array}{l}\text { rise time } \\
(\tau)\end{array}$ & $\begin{array}{l}\text { current peak } \\
\text { (A) }\end{array}$ & $\begin{array}{c}\text { current peak } \\
(A)\end{array}$ \\
\hline & & $50 \mu \mathrm{m}$ & 11.095 & 0.0040532 & $-9.7153 \times 10^{-6}$ \\
\hline$\varepsilon_{2}=\varepsilon_{1}=3.9 \varepsilon_{0}$ & , & $5 \mu m$ & 12.040 & 0.0046107 & -0.0012016 \\
\hline$h_{2}=2.5 \mu \mathrm{m}$ & & $0.5 \mu \mathrm{m}$ & 15.673 & 0.0069098 & -0.0013227 \\
\hline & & $0.05 \mu \mathrm{m}$ & 35.087 & 0.0144630 & -0.00069220 \\
\hline$\varepsilon_{1}=3.9 \varepsilon_{0}$ & & $10 \times \varepsilon_{1}$ & 15.316 & 0.0064447 & -0.0011712 \\
\hline & $\varepsilon_{2}$ & $\varepsilon_{1}$ & 12.739 & 0.0050056 & -0.0013332 \\
\hline$h_{2}=0.5 \mu m$ & & $0.1 \times \varepsilon_{1}$ & 11.095 & 0.0042048 & -0.0014120 \\
\hline
\end{tabular}

the rise time decreases at the same time but shows slower as the $h_{1}$ grows larger. During the four cases in rows 3-6, the current peak of $M_{2}$ varies dramatically. The portion of the current flowing to $M_{2}$ reaches a maximum value of one-quarter of the $M_{1}$ total current when $h_{1}$ is $5 \mu \mathrm{m}$ and $M_{1}$ is the second nearby case to $M_{2}$ in the four cases. It is because that as $h_{1}$ changes from 0.5 to $5 \mu \mathrm{m}$, the current flowing to the ground plane decreases faster than current flowing to $M_{2}$. However, the larger coupling capacitance results in the larger transient current to $M_{2}$ conductor and at the same time the larger voltage response of cross talk occurs. Furthermore, as $M_{1}$ is far from $M_{2}$, the rise time reaches saturation value 11.095 shown in row 3, which is almost independent of $M_{2}$ and ground plane. Another interesting case investigates the influence of different permittivities of multilayers shown in rows 7-9. One can see that as the permittivity $\varepsilon_{2}$ increases exponentially from 0.1 to $10 \varepsilon_{1}$, the peak current of $M_{1}$ increases linearly and the current flow to $M_{2}$ is almost unchanged around 0.001, which shows quite different behaviors from case 1 . It is obvious that the rise time of $M_{1}$ shows linear dependence on the current peak of $M_{1}$. Especially, due to the larger permittivity in the region 2 , the larger electric field flux flowing from the top of $M_{1}$ conductor to the ground plane and the slower rising time of voltage for $M_{1}$ conductor result in the larger ground capacitance. From the above analysis, it's important to incorporate 
the consideration of multilayer topography for modeling the transient characteristics of multilevel interconnection.

\section{CONCLUSIONS}

To model the transient characteristics of distributed resistorcapacitor of ULSI multilevel interconnections on complex topography, we have proposed an efficient method to avoid the redundant works on both volume mesh and transient analysis associated with the finite-difference method. The timedependent boundary-element integral formulation associated with the PVL algorithm is first introduced to analyze efficiently the transient characteristics for arbitrary configurations of multilevel conductor system on complex topography. An adaptive multilayer Green's function is adopted to investigate several cases that have revealed interesting physical mechanisms in charge transfer between conductors on multilayer topography. The actual topography of multilevel conductor system becomes more realistic in modeling its transient characteristics. The formulations of poles and residues are derived, which can be calculated fast enough to be easily included in a circuit simulator. Hence it is easy to calculate the transient characteristics of both parallel conductors and complicated configurations such as crossing lines, corners, contacts, multilayers and their combinations.

\section{REFERENCES}

[1] L. T. Pillage and R. A. Rohrer, "Asymptotic waveform evaluation for timing analysis," IEEE Trans. Computer-Aided Design, vol. 9, p. 352, Apr. 1990.

[2] S. Kumashiro, R. A. Rohrer, and A. J. Strojwas, "Asymptotic waveform evaluation for transient analysis of 3-D interconnect structures," IEEE Trans. Computer-Aided Design, vol. 12, p. 988, July 1993.

[3] H. M. Hou, C. S. Sheen, and C. Y. Wu, "A novel modeling technique for efficiently computing 3-D capacitances of VLSI multilevel interconnections-BFEM," IEEE Trans. Electron Devices, vol. 45, p. 200, Jan. 1998.

[4] D. D. Ling, S. Kim, and J. White, "A boundary-element approach to transient simulation of three-dimensional integrated circuit interconnect," in Proc. 29th Design Automation Conf., 1992, p. 93.

[5] P. Feldmann and R. W. Freund, "Efficient linear circuit analysis by Padè approximation via the Lanczos process," IEEE Trans. Computer-Aided Design, vol. 14, p. 639, May 1995

[6] T. Fukui, T. Fukuhara, and T. Furuichi, "Three-dimensional analysis of a fresh water lens in an island," in Proc. U.S. Japan Seminar on BEM: Boundary Element Methods in Applied Mechanics, 1988, p. 285.

[7] Z. Q. Ning, P. M. Dewilde, and F. L. Neerhoff, "Capacitance coefficients for VLSI multilevel metallization lines," IEEE Trans. Electron Devices, vol. ED-34, p. 644, Mar. 1987.

[8] A. E. Ruehli and P. A. Brennan, "Capacitance model for integrated circuit metallization wires," IEEE J. Solid-State Circuits, vol. SC-10, p. 530, Dec. 1975.

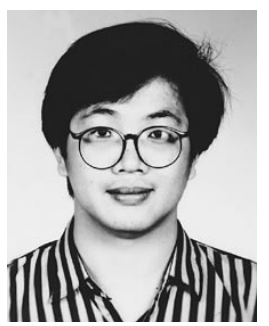

Hsin-Ming Hou (S'92) was born in Tainan, Taiwan, R.O.C., on December 29, 1968. He received the B.S. degree from the Physics Department, National Chang Hua Normal University, Changhua, Taiwan, in 1990, the M.S. degree from the Institutes of Electro-Physics, in 1992, and the Ph.D. degree from the Institute of Electronics, in 1998. both from National Chiao-Tung University, Hsinchu, Taiwan,

$\mathrm{He}$ is serving in the R.O.C. Army as a military policeman. His specialties include computer-aided simulation of electronic circuits and device modeling.

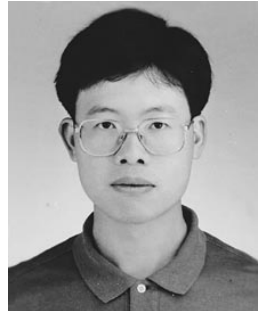

Chin-Shown Sheen (S'92) received the B.S. degree from the Physics Department, National Chang Hua Normal University, Changhua, Taiwan, R.O.C., and the M.S. degree from the Institute of Physics, National Tsing Hwa University, Hsinchu, Taiwan, in 1998 and 1990, respectively. He is currently pursuing the Ph.D. degree at the Institute of ElectroOptical Engineering, National Chiao-Tung University, Hsinchu. His current research area is in sensor simulation and device modeling.

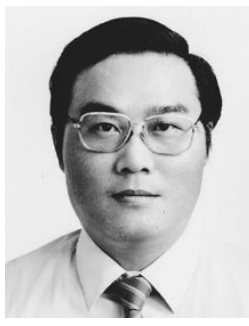

Chin-Yuan Wu (M'72) was born in Taiwan, R.O.C., on March 18, 1946. He received the B.S. degree from the Department of Electrical Engineering, National Taiwan University, Taipei, in 1968, and the M.S. and Ph.D. degrees from the State University of New York (SUNY), Stony Brook, in 1970 and 1972, respectively.

During the 1972-1973 academic year, he was appointed as a Lecturer at the Department of Electrical Sciences, SUNY. From 1973 to 1975 , he was a Visiting Associate Professor at National Chiao-Tung University (NCTU), Hsinchu, Taiwan. In 1976, he became a Full Professor in the Department of Electronics and the Institute of Electronics, NCTU. At NCTU, he was the Director of Engineering Laboratories and Semiconductor Research Center from 1974 to 1980; the Director of the Institute of Electronics from 1978 to 1984; and the Dean, College of Engineering, from 1984 to 1990 . He was a Principal Investigator of the National Electronics Mass Plan-Semiconductor Devices and IntegratedCircuit Technologies from 1976 to 1979, and was a Coordinator of the National Microelectronics Researches and High-Level Man-Power Education Committee, National Science Council, R.O.C., from 1982 to 1988. He has been the Research Consultant for the Electronics Research and Service Organization (ERSO), ITRI; a member of the Academic Review Committee, the Ministry of Education; and the Chairman of the Technical Review Committee on Information and Microelectronics Technologies, the Ministry of Economic Affairs. His research activities have been in semiconductor device physics and modelings, and integrated-circuit designs and technologies. His present research areas focus on the developments of efficient 2-D and 3-D simulators for deep-submicrometer semiconductor devices, design rules and optimization techniques for deep-submicrometer CMOS devices, and key technologies for deep-submicrometer CMOS devices. He has published more than 180 papers in the semiconductor field and has served as a reviewer for international journals such as IEEE Electron Device LeTters, IEEE Transactions on Electron Devices, and Solid-State Electronics.

Dr. Wu is a member of the Honorary Editorial Advisory Board of SolidState Electronics. He received the Academic Research Award in Engineering from the Ministry of Education (MOE) in 1979, and the Outstanding Scholar Award from the Chinese Educational and Cultural Foundation in 1985. He received the Outstanding Research Professor Fellowship from the Ministry of Education and the National Science Council (NSC), R.O.C., from 1982 to 1999. He received the Distinguished Engineering Professor Medal Award from the Chinese Engineering Society in 1992. 